\title{
ABI4 mediates antagonistic effects of abscisic acid and gibberellins at transcript and protein levels
}

Kai Shu ${ }^{1,2, \dagger}$, Qian Chen ${ }^{2,3, \dagger}$, Yaorong Wu ${ }^{2}$, Ruijun Liu ${ }^{2,3}$, Huawei Zhang ${ }^{2}$, Pengfei Wang ${ }^{2}$, Yanli Li' ${ }^{2}$, Shengfu Wang ${ }^{2}$, Sanyuan Tang ${ }^{2}$, Chunyan Liu ${ }^{2}$, Wenyu Yang ${ }^{1}$, Xiaofeng $\mathrm{Cao}^{2}$, Giovanna Serino ${ }^{4, *}$ and $\mathrm{Qi} \mathrm{Xie}^{2, *}$

${ }^{1}$ Key Laboratory of Crop Ecophysiology and Farming System in Southwest China, Institute of Ecological Agriculture, Sichuan Agricultural University, Chengdu 611130, China,

${ }^{2}$ State Key Laboratory of Plant Genomics, Institute of Genetics and Developmental Biology, Chinese Academy of Sciences, Beijing 100101, China,

${ }^{3}$ University of Chinese Academy of Sciences, Beijing 100049, China, and

${ }^{4}$ Department of Biology and Biotechnology 'Charles Darwin', Sapienza University, Rome 00185, Italy

Received 15 February 2015; revised 4 December 2015; accepted 7 December 2015; published online 28 December 2015.

*For correspondence (e-mail qxie@genetics.ac.cn; giovanna.serino@uniroma1.it).

TThese authors contributed equally to this work.

\begin{abstract}
SUMMARY
Abscisic acid (ABA) and gibberellins (GAs) are plant hormones which antagonistically mediate numerous physiological processes, and their optimal balance is essential for normal plant development. However, the molecular mechanism underlying $A B A$ and $G A$ antagonism still needs to be determined. Here, we report that ABA-INSENSITIVE 4 (ABI4) is a central factor in GA/ABA homeostasis and antagonism in post-germination stages. $A B I 4$ overexpression in Arabidopsis (OE-ABI4) leads to developmental defects including a decrease in plant height and poor seed production. The transcription of a key $A B A$ biosynthetic gene, NCED6, and of a key GA catabolic gene, GA20x7, is significantly enhanced by $A B \mid 4$ overexpression. ABI4 activates NCED6 and GA20x7 transcription by directly binding to the promoters, and genetic analysis revealed that mutation in these two genes partially rescues the dwarf phenotype of $A B I 4$ overexpressing plants. Consistently, $A B I 4$ overexpressing seedlings have a lower GA/ABA ratio than the wild type. We further show that ABA induces GA2ox7 transcription while GA represses NCED6 expression in an ABI4-dependent manner; and that $A B A$ stabilizes the $A B I 4$ protein whereas GA promotes its degradation. Taken together, these results suggest that $A B A$ and $G A$ antagonize each other by oppositely acting on $A B I 4$ transcript and protein levels.
\end{abstract}

Keywords: ABA, GA, ABI4, antagonism, transcription factor.

\section{INTRODUCTION}

Plant growth and development is the end result of the interaction of diverse endogenous signals with environmental cues (Rymen and Sugimoto, 2012). Each phytohormone acts at low concentrations to regulate numerous aspects of plant development, with distinct or synergistic functions (Gray, 2004; Vanstraelen and Benkova, 2012). Many elegant studies have demonstrated that optimal hormone levels are essential for the achievement of plant normal growth and development (Lee et al., 2002, 2012; Porri et al., 2012).

Gibberellins (GAs) are a large group of tetracyclic diterpenoid plant hormones which regulate diverse developmental processes throughout the plant life cycle, including seed germination, stem elongation, leaf expansion, trichome and root development, and the transition from vegetative growth to reproductive growth (Yamaguchi, 2008; Nelissen et al., 2012; Porri et al., 2012). A number of genes have been implicated in the GA metabolism pathway: bioactive GAs are synthesized by GA3 and GA20 oxidases (GA3ox and GA20ox) and catabolized by a group of catabolic GA2 oxidases (GA2ox) (Rieu et al., 2008b; Porri et al., 2012). In line with these findings, plants overexpressing GA2ox genes have a reduced content of bioactive GAs and show GA-deficient phenotypes similar to those displayed by mutants deficient in GA biosynthesis (Lee et al., 2002; Schomburg et al., 2003; Magome et al., 2008; Rieu et al., 2008a; Porri et al., 2012). These phenotypes include dwarf or semi-dwarf stature (Magome et al., 2008; Porri et al., 
2012). On the contrary, plants with an elevated GA content have, among other defects, a taller stature (Busov et al., 2008). Therefore, the endogenous GA level must be precisely regulated in order to achieve proper plant development.

Gibberellins positively regulate plant growth by promoting the degradation of a group of DELLA proteins, which inhibit plant growth and act as negative regulators in the GA signaling pathway (Peng et al., 1997; Lee et al., 2002, 2012; Feng et al., 2008). The Arabidopsis genome encodes five DELLA proteins: GA INSENSITIVE (GAI), REPRESSOR OF GA1-3 (RGA), RGA-LIKE1 (RGL1), RGL2, and RGL3, which possess distinct or synergistic biological functions during different stages of plant development (Lee et al., 2002; Cheng et al., 2004; Piskurewicz and Lopez-Molina, 2009). The Arabidopsis ubiquitin ligase complexes SCF ${ }^{\mathrm{SLY} 1}$ and SCF $^{\text {SNE1 }}$ target the DELLA protein(s) for $26 \mathrm{~S}$ proteasome-mediated degradation, and the interaction between GA and its receptor GID1 promotes degradation of DELLAs (Ueguchi-Tanaka et al., 2005; Willige et al., 2007; Murase et al., 2008).

Abscisic acid ( $A B A)$ regulates a variety of developmental processes including seed dormancy and germination, root development, stomatal movement and adaptive stresses responses (Nambara and Marion-Poll, 2005; Cutler et al., 2010). Extensive studies have demonstrated that ABA generally regulates development by retarding plant growth (Barrero et al., 2005; Nambara and Marion-Poll, 2005; Fujii and Zhu, 2009; Cutler et al., 2010). Consistently, a constitutively elevated ABA level in plant causes severe growth defects (Fan et al., 2009). Therefore it is not surprising that, similar to GAs, the level of endogenous active ABA must be strictly determined by tight control of its rate of biosynthesis and catabolism (Nambara and Marion-Poll, 2005).

Abscisic acid and GA antagonistically regulate many physiological processes including seed germination and plant growth, as well as their own metabolic processes. A Iow ABA level promotes GA biosynthesis (Seo et al., 2006) and vice versa (Oh et al., 2007). However, the precise molecular mechanism by which $A B A$ and GA antagonize each other has long eluded researchers. Nevertheless, several factors have been isolated which might mediate - at least partially - the antagonistic effects of these two hormones. For example, GA has been shown to inhibit $A B A$ biogenesis by repressing the expression of XERICO, which encodes an E3 ubiquitin ligase that enhances ABA biosynthesis by promoting the expression of the ABA biosynthesis gene NCED3 (Ko et al., 2006; Zentella et al., 2007). In addition, the transcription factor FUS3 has been shown to inhibit GA biosynthesis by directly binding to the GA3ox2 promoter, while promoting the accumulation of $A B A$ with an as yet uncharacterized mechanism (Curaba et al., 2004; Gazzarrini et al., 2004). Recently, the transcription factor OsAP2-39 was demonstrated to play a key role in mediat- ing the GA/ABA balance in rice (Yaish et al., 2010). Several studies also point to a key role of an APETALA2 (AP2) family member, ABA-INSENSITIVE 4 (ABI4), in the control of GA/ABA homeostasis: we have recently shown that $A B I 4$ controls primary seed dormancy by regulating the balance between ABA and GA metabolism (Shu et al., 2013). In addition, we and others have shown that $A B I 4$, by positively regulating $A B A$ signaling, is also involved in the control of other aspects of plant development in addition to seed dormancy and germination (Finkelstein, 1994; Finkelstein et al., 1998; Soderman et al., 2000; Shu et al., 2013). These novel aspects include lipid mobilization from the embryo (Penfield et al., 2006), glucose signaling (ArenasHuertero et al., 2000; Laby et al., 2000), the salt stress response (Quesada et al., 2000), regulation of plant male sterility (Shu et al., 2014) and the mitochondrial and chloroplast-nucleus retrograde signaling pathways (Koussevitzky et al., 2007; Giraud et al., 2009; Sun et al., 2011). Recently, ABI4 was also shown to regulate ABA and cytokinin-mediated inhibition of lateral roots by impairing polar auxin transport (Shkolnik-Inbar and Bar-Zvi, 2010). Furthermore, ABI4 is downstream of both ABA- and jasmonic acid (JA)-dependent signaling pathways (Kerchev et al., 2011), and mediates plant responses to both sugar and ABA signaling (Li et al., 2014). Therefore ABI4 seems to be a highly versatile factor which may function in diverse signaling pathways.

Here, we report that $A B I 4$ is a key factor in the modulation of GA/ABA homeostasis and antagonism. We show that ectopic expression of $A B / 4(O E-A B / 4)$ leads to pleiotropic phenotypic defects including dwarf stature and poor seed production. $O E-A B / 4$ lines have a lower GA/ABA ratio than the wild type, and $A B I 4$ directly promotes the expression of the ABA biosynthetic gene NCED6 and the GA catabolic gene GA2ox7. In line with these results, mutations in nced 6 and ga2ox 7 can partially rescue the dwarf phenotype of $O E-A B / 4$. Furthermore, $A B A-m e d i a t e d$ induction of GA2Ox7 and GA-mediated inhibition of NCED6 both depend on $A B I 4$. At the protein level, ABA stabilizes $A B I 4$ whereas GA promotes its degradation. Taken together, our results suggest that $A B I 4$ is not only a key regulator of $G A / A B A$ homeostasis but also a key target of GA/ABA antagonism.

\section{RESULTS}

\section{Overexpression of $A B I 4$ causes pleiotropic phenotypes}

A previous study demonstrated that $A B / 4$ is expressed at higher levels in maturing and germinating seeds and at lower levels in almost all tissues during vegetative growth (Soderman et al., 2000) and in previous work we have shown that $A B I 4$ regulates seed dormancy (Shu et al., 2013). In this work we further dissect the role of $A B I 4$ in post-germination stages. We took advantage of a gain-offunction approach and analyzed the phenotypes due to 
$A B / 4$ overexpression. We used the same two independent $O E-A B / 4$ transgenic lines (named OE1 and OE2) that we employed in our previous work (Shu et al., 2013) and compared their phenotypes with those of the abi4 mutant (Finkelstein, 1994; Shu et al., 2013).

At an early growth stage (6-day-old seedlings), both abi4 and $O E-A B I 4$ plants showed abnormal leaf size: the true leaves were slightly larger in abi4 seedlings and significantly smaller in $O E-A B / 4$ seedlings when compared with the wild-type (WT) control (Figure 1a and Figure S1 in the Supporting Information). abi4 mutant plants had no detectable defects at later growth stages, as also indicated by previous reports (Soderman et al., 2000). On the contrary, adult $O E-A B I 4$ plants showed pleiotropic defects, including shorter petioles, smaller rosettes and fewer and shorter siliques, and consequently reduced seed production (Figure 1). $O E-A B / 4$ plants were also dwarf and much shorter than the WT control. In detail, in $O E-A B / 4$ plants, height was reduced by $43-67 \%$, rosette size by $58-68 \%$ and the silique number per plant by $15-30 \%$ when compared with the WT (Figure S1). The seed yield of the transgenic plants was about $20-26 \%$ of the WT yield (Figure S1), and since the 1000-grain weight was not significantly affected by $A B I 4$ overexpression, the difference in seed yield was most likely due to a reduction in seed number rather than seed size (Figures S1 and S2). These defects were not detected in other $O E-A B / 4$ transgenic lines with lower $A B / 4$ expression levels, suggesting that they are due to $A B / 4$ overexpression, and that strict regulation of $A B / 4$ transcription is essential for normal plant development.

\section{$A B \mid 4$ affects the transcription of several $A B A$ and $G A$ metabolism genes}

The dwarf phenotype of $O E-A B / 4$ lines is reminiscent of the phenotypes of plants with defects in the $G A$ or $A B A$ pathways, suggesting that GA and $A B A$ metabolism and/or signaling might be altered in these lines (Fan et al., 2009; Porri et al., 2012).

To explore this possibility, we first examined the expression of selected GA catabolic genes (GA20ox2 and GA2ox7) in the two $O E-A B I 4$ lines, the abi4 mutant and their WT control by quantitative reverse transcription PCR (qRT-PCR) using two reference genes ( $18 S$ and $A C T I N)$. Only genes showing a significant result with both normalizers and, in the case of $O E-A B I 4$, in both overexpressing lines, were considered to be differentially expressed. While GA20ox2 expression was not changed in the overexpressing lines according to our criterion (Figures $2 \mathrm{a}$ and S4a), GA2ox7 was significantly upregulated in both $O E-A B / 4$ lines (Figures $2 \mathrm{~b}$ and $\mathrm{S} 4 \mathrm{~b})$. In particular the GA2ox7 transcript level was 5 -fold and 26-fold higher than the WT in OE-1 and $\mathrm{OE}-2$, respectively. On the contrary, these genes were not (a)
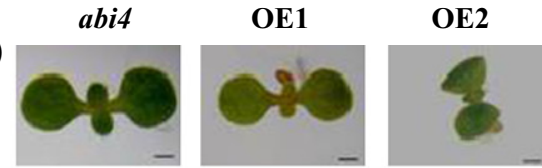

WT

(b)
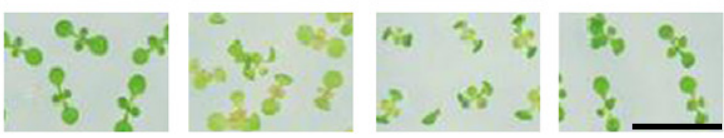

(c)

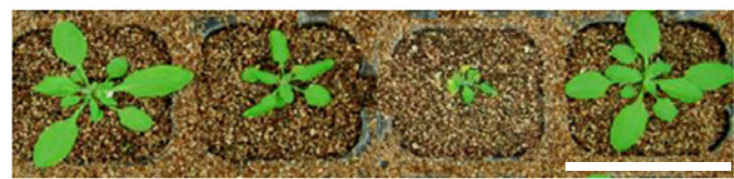

(d)

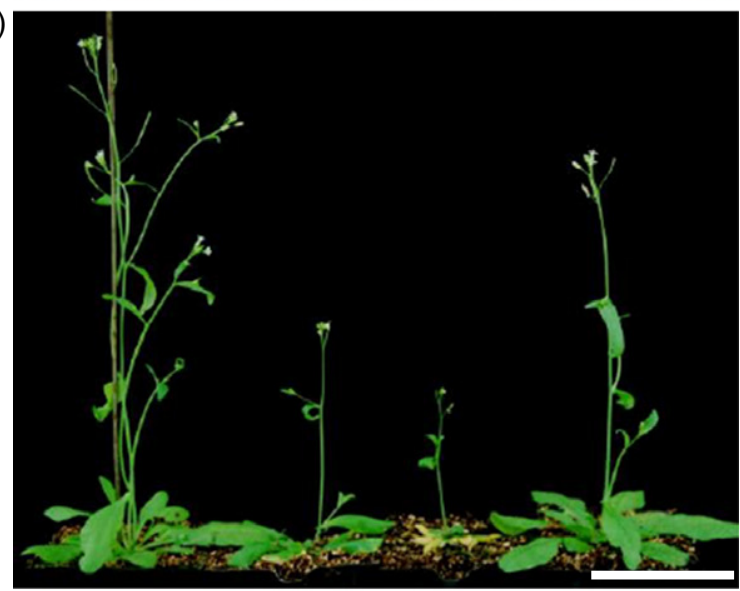

(e)

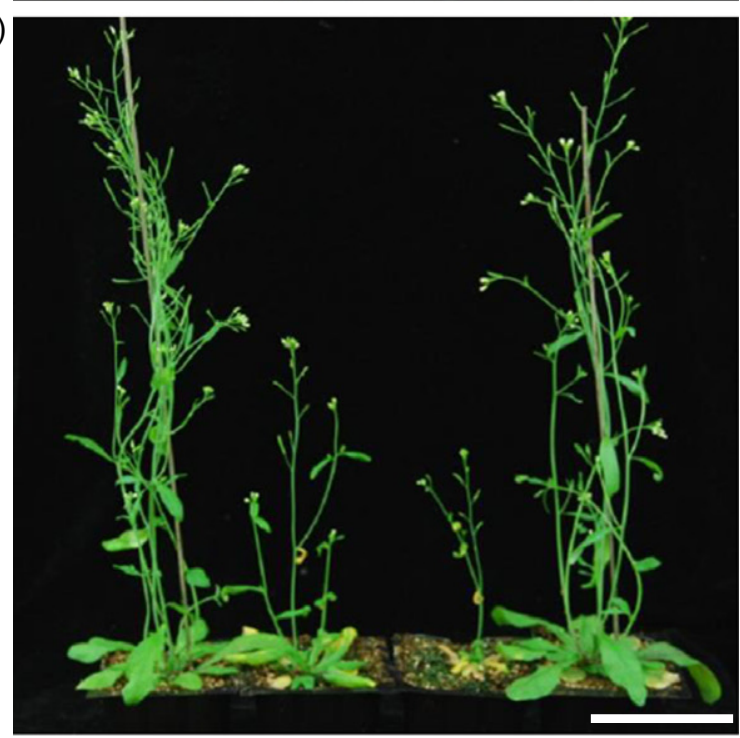

Figure 1. Post-germination phenotypes of abi4, wild-type (WT) and OE $A B / 4$ plants at different developmental stages.

$\mathrm{OE} 1$ and $\mathrm{OE} 2$ represent two independent $O E-A B / 4$ transgenic lines.

(a) Six-day-old seedlings, bar $=0.5 \mathrm{~mm}$.

(b) Ten-day-old seedlings, bar $=5 \mathrm{~mm}$.

(c) Four-week-old plants, bar $=20 \mathrm{~mm}$.

(d) Five-week-old plants, bar $=20 \mathrm{~mm}$.

(e) Seven-week-old plants, bar $=20 \mathrm{~mm}$. 


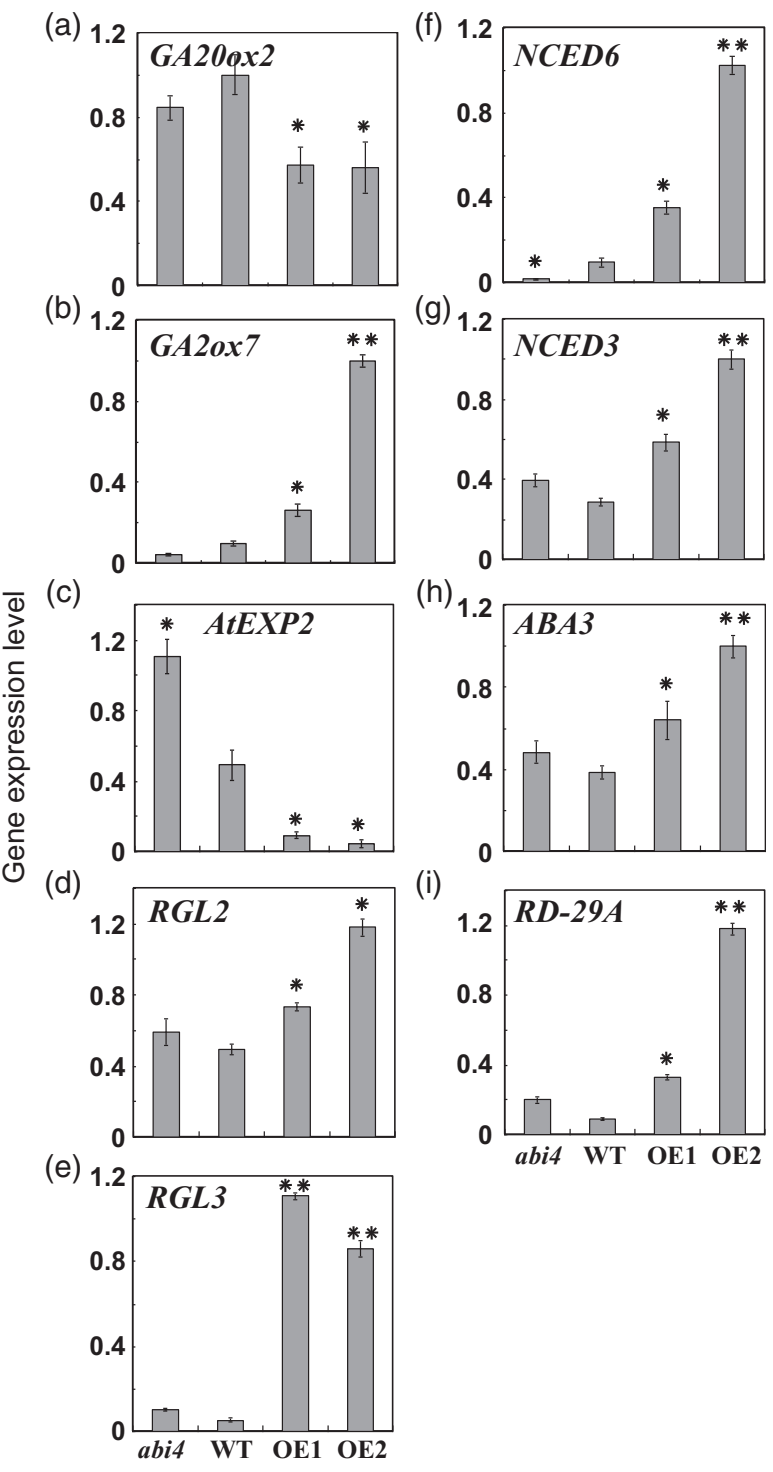

Figure 2. $A B I 4$ regulates the transcription profiles of specific gibberellin (GA) and ABA metabolism genes.

Quantitative RT-PCR analysis of GA20ox2 (a), GA2Ox7 (b), AtEXP2 (c), RGL2 (d), RGL3 (e), NCED6 (f), NCED3 (g), ABA3 (h) and RD-29A (i), in 2-week-old abi4, $O E-A B / 4$ and wild-type (WT) seedlings.

$\mathrm{OE} 1$ and $\mathrm{OE} 2$ represent two independent $O E-A B / 4$ transgenic lines. The $18 S$ rRNA was used as the reference gene. The experiments were performed in three replicates and one typical experiment is shown. Asterisks indicate statistically significant differences from WT $(* P<0.05 ; * * P<0.01)$

significantly affected in the abi4 mutant (Figures 2a,b and $\mathrm{S} 4 \mathrm{a}, \mathrm{b})$, consistent with the weak phenotype of these mutant seedlings. However, these results, together with our finding that abi4 seedlings showed instead a significant increase in the expression of GA20ox1 with both normalizers (Figures $\mathrm{S} 3 \mathrm{a}$ and $\mathrm{S} 4 \mathrm{i}$ ), suggest that the endogenous $\mathrm{GA}$ level might be altered in $O E-A B / 4$ transgenic plants.

To test this hypothesis, we analyzed the expression level of genes known to be regulated by GA, such as AtEXP2,
RGL2 and RGL3 (Lee et al., 2010; Yamauchi et al., 2004). Indeed, while the AtEXP2 transcript level was decreased in $O E-A B / 4$ plants when compared with the WT (Figure 2c), the level of expression of $R G L 2$ and $R G L 3$ was significantly higher in $O E-A B I 4$ plants than in the WT. Expression of these genes, however, was not significantly altered in the abi4 mutant (Figures $2 \mathrm{c}-\mathrm{e}$ and $\mathrm{S} 4 \mathrm{c}, \mathrm{d}$ ), again consistent with the weak phenotype of these mutants. Taken together, these results further strengthened our hypothesis that $O E$ $A B / 4$ plants might contain a reduced amount of active GA, and that this might be responsible for the differential expression of GA-regulated genes.

Given the antagonistic effect of $A B A$ and $G A$ in the control of plant growth and development (Vanstraelen and Benkova, 2012), we also investigated whether $A B / 4$ overexpression affected the expression of selected genes involved in ABA anabolism, catabolism or response pathways. As reported in Figures $2(f-h)$ and $S 4(e-g)$, the transcript levels of the ABA biosynthesis genes NCED6, NCED3 and $A B A 3$ were significantly increased in $O E$ $A B I 4$ plants compared with the WT (Figures $2 \mathrm{f}-\mathrm{h}$ and $\mathrm{S} 4 \mathrm{e}-\mathrm{g}$ ). The level of transcription of NCED6 in particular was not only remarkably enhanced in the $O E-A B / 4$ lines but also decreased in the abi4 mutant (Figures $2 f$ and $\mathrm{S} 4 \mathrm{e})$. Furthermore, to our surprise, the expression of the ABA catabolic genes CYP707A2 and CYP707A3 was also significantly upregulated in $O E-A B I 4$ seedlings (Figure $S 3 b, c)$, and, in the case of CYP707A2, downregulated in the abi4 mutant, further indicating that ABA metabolism might be altered by non-physiological levels of $A B / 4$.

To address whether the altered expression of $A B A$ metabolism genes in $O E-A B I 4$ plants also affected the $A B A$ response, we monitored the expression of $R D 29 A$, an $A B A$-inducible gene that contains $A B A$-responsive elements in its promoter (Shinozaki and Yamaguchi Shinozaki, 1997; Xiong et al., 2001), in $O E-A B / 4$ transgenic lines. While no significant difference in $R D 29 A$ expression was observed between the abi4 mutant and the WT, the RD29A transcript level was significantly increased in $O E-A B / 4$ (Figures $2 \mathrm{i}$ and $\mathrm{S} 4 \mathrm{~h}$ ) when compared with the WT, suggesting that the complex expression profile of genes involved in ABA catabolism and biosynthesis might result in a higher $A B A$ content in $O E-A B / 4$ plants, which in turn could lead to enhanced expression of RD29A.

\section{$A B I 4$ enhances $A B A$ biosynthesis and GA catabolism in seedlings}

To confirm the hypothesis that $O E-A B / 4$ might contain abnormal levels of $G A$ and $A B A$, we next measured the endogenous GA and $A B A$ content using our previously reported assay (Chen et al., 2011; Shu et al., 2013). Because of the phenotypic similarity between the two $O E-A B / 4$ lines (Figures 1, 2 and $\mathrm{S} 1$ ), only one line (OE1) was chosen for these experiments. Consistent with our 
(a)
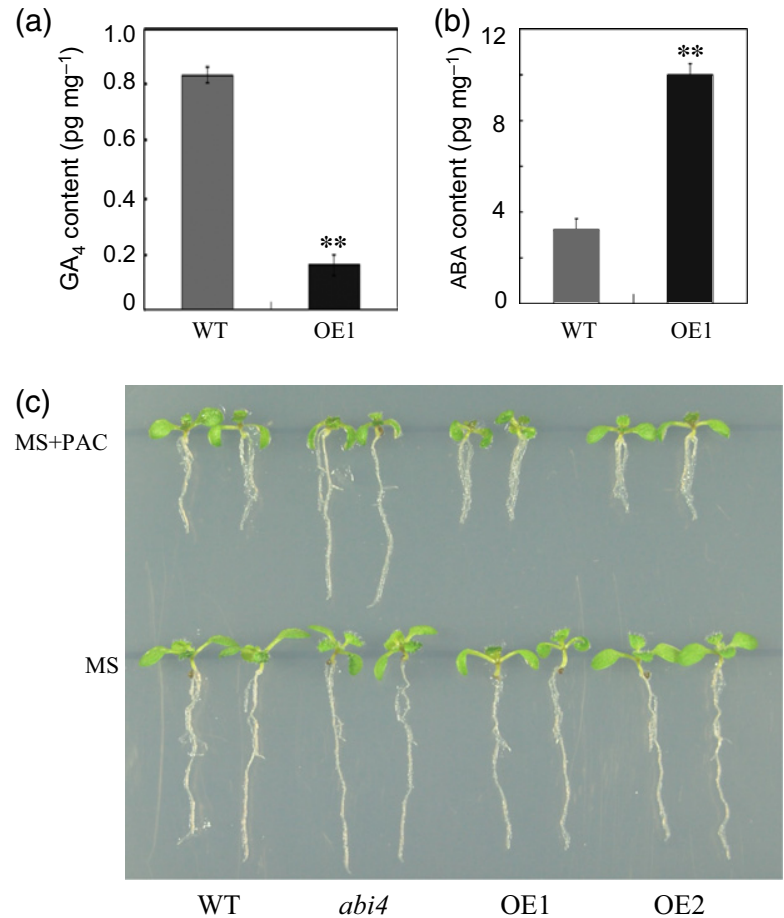

Figure 3. $A B / 4$ overexpressing lines have a lower gibberellin (GA) and higher $A B A$ content.

(a) The GA content of 2-week-old wild-type (WT) and OE-AB/4 line 1 (OE1) seedlings.

(b) The ABA content of 2-week-old WT and OE-AB/4 line 1 (OE1) seedlings. (c) Phenotypic comparison of 2-week-old abi4, WT and $O E-A B / 4$ seedlings grown in the presence (top) or absence (bottom) of paclobutrazol (PAC). $\mathrm{OE} 1$ and $\mathrm{OE} 2$ represent two independent $O E-A B / 4$ transgenic lines. Asterisks indicate statistically significant differences between $O E-A B / 4$ and WT $(* * P<0.01)$.

hypotheses, the GA content was lower and the $A B A$ content higher in the $O E-A B / 4$ plants than in the WT (Figure $3 a, b)$. To further confirm the GA measurement results, we analyzed the root growth pattern of abi4 and $O E-A B / 4$ seedlings in response to paclobutrazol (PAC), an inhibitor of $\mathrm{GA}$ biosynthesis. Our results showed that while root growth of $O E-A B / 4$ seedlings was slightly sensitive to PAC treatment, abi4 seedlings were resistant to PAC (Figure 3c). Because it has been shown that seedlings with a higher GA content are more resistant to PAC and seedlings with lower GA content are more sensitive to it (Zhang et al., 2011b), our results are consistent with a lower GA content in $O E-A B / 4$ lines (Figure 3a).

We also hypothesized that the higher $A B A$ level in $O E$ $A B / 4$ plants might be the cause of the upregulation of the catabolic genes CYP707A3 and CYP707A2 (18S for Figure S3b,c; ACTIN for Figure S4j,k). This upregulation could be due to feedback regulation by the higher ABA content in these plants. Indeed, information retrieved from the public Arabidopsis microarray database (http://bbc.botany.utoronto.ca/efp/cgi-bin/efpWeb.cgi) revealed that exoge- nous $A B A$ treatment strongly induces transcription of CYP707A2 (Figure S5a) but has no obvious effect on CYP707A3 expression (Figure S5b), and our qRT-PCR analysis (Figure S5c,d) showed that CYP707A2 at least is progressively upregulated by an increasing concentration of exogenous ABA. Similar qRT-PCR results were also obtained when the ACTIN reference gene was employed (Figure S5e,f).

\section{ABI4 directly binds to the GA2ox7 and NCED6 promoters in vivo}

$\mathrm{ABI} 4$ is an $\mathrm{AP} 2$-domain-containing transcription factor, and previous studies have demonstrated that $A B I 4$ regulates gene expression by binding to a CCAC motif within the promoters of target genes (Acevedo-Hernandez et al. 2005; Koussevitzky et al., 2007). Because ABI4 overexpression leads to misexpression of a series of $A B A$ and $G A$ metabolism genes, we investigated whether $A B I 4$ directly regulates the transcription of these genes. First, we analyzed the promoter sequences of the genes whose transcription is under $\mathrm{ABI} 4$ control (Figures 2 and S3). We found that the promoters of both GA2ox7 and NCED6 contain putative $A B \mid 4-b i n d i n g$ motifs: the $G A 20 \times 7$ promoter contains 5 CCAC motifs while the NCED6 promoter contains 10 motifs (Figure 4a,b), indicating that $A B \mid 4$ might directly bind to these promoters. However, another transcription factor, DDF1, is known to directly bind to the GA2ox7 promoter and enhance its expression (Magome et al., 2008). Thus, to exclude the possibility that $A B I 4$ regulates $G A 20 x 7$ transcription indirectly by affecting $D D F 1$ expression, we analyzed the DDF1 transcript level in the abi4 and $O E-A B / 4$ lines and did not observe any noticeable change (Figure S6).

We next tested if $A B I 4$ binds directly to the GA2ox7 and NCED6 promoters by performing chromatin immunoprecipitation (ChIP)-qPCR assays on a 35S-AB/4-GFP transgenic line (Shu et al., 2013). The $A B / 5$ promoter was used as a positive control since it was already known to be directly bound by ABI4 (Bossi et al., 2009). Protein and DNA complexes were immunoprecipitated using an antibody against the GFP, and enriched DNA was amplified by qPCR using specific primers sets that anneal to the CCAC motifs (P1-3 on GA2ox7; P5-8 on NCED6) or to CCAC-poor regions (P4 on GA2ox7) present in the GA2ox7 and NCED6 promoters (Figure 4a,b). As shown in Figure 4(c), we found enrichment of the P1 and P3 regions of the GA2ox7 promoter and of the P5 and P7 regions of the NCED6 promoter, suggesting that GA2ox7 and NCED6 are direct targets of $A B \mid 4$. Similar results were obtained using two independent 35S-ABI4-GFP transgenic lines, which indicated that $\mathrm{ABI} 4$ promotes GA20x7 and NCED6 transcription by directly binding to their promoters. However, we did not detect an enrichment of ABI4 on ABA3 and NCED3 promoters, although they also contained a series of CCAC 
Figure 4. $A B \mid 4$ binds directly to the $G A 20 x 7$ and NCED6 promoters in vivo.

(a), (b) Schematic representation of the GA20x7 (a) and the NCED6 (b) promoters. Black boxes indicate the position of the CCAC motif. The arrows indicate the positions of the primers used in part (c).

(c) Chromatin immunoprecipitation-quantitative PCR analysis conducted using the specific primers pairs indicated in (a). TUB4 was used as an internal control and a specific region of the $A B / 5$ promoter as a positive control. The experiments were conducted in three replicates on two independent 35SABI4-GFP lines (OE1 and OE2), and one typical experiment result is shown.

(d), (e) Histochemical assay (d) and quantitative analysis (e) of $N$. benthamiana leaves transformed with the constructs indicated in the figure. (d) Representative GUS-staining images of samples taken from $N$. benthamiana leaves at 3 days after infiltration. (e) Quantitative analysis of relative GUS activity from samples taken from the same leaves shown in (d). Activity units are given in $\mathrm{nmol}$ methyl-umbelliferone (mg protein $)^{-1} \mathrm{~min}^{-1}$. The experiments were performed in three biological replicates and one typical experiment is shown. Asterisks indicate statistically significant differences between HA-GFP- and ABI4-GFP-transformed leaves $(* P<0.05)$. (a)

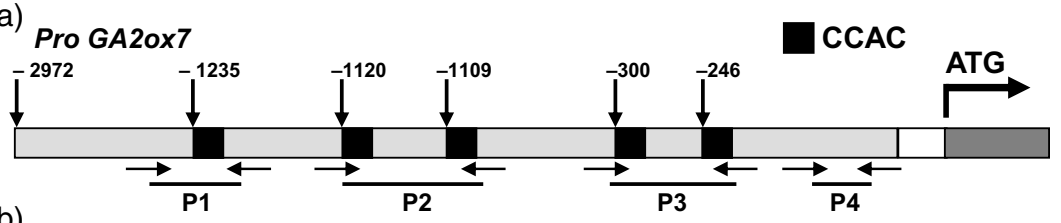

(b)

\section{Pro NCED6}

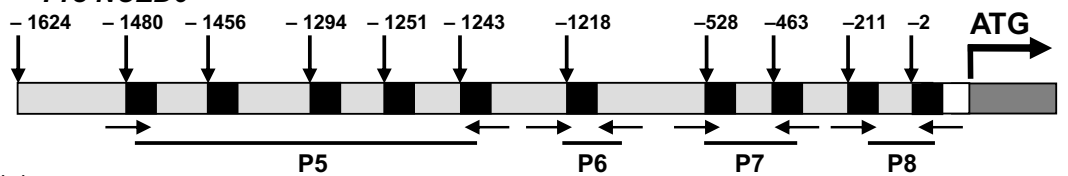

(c)

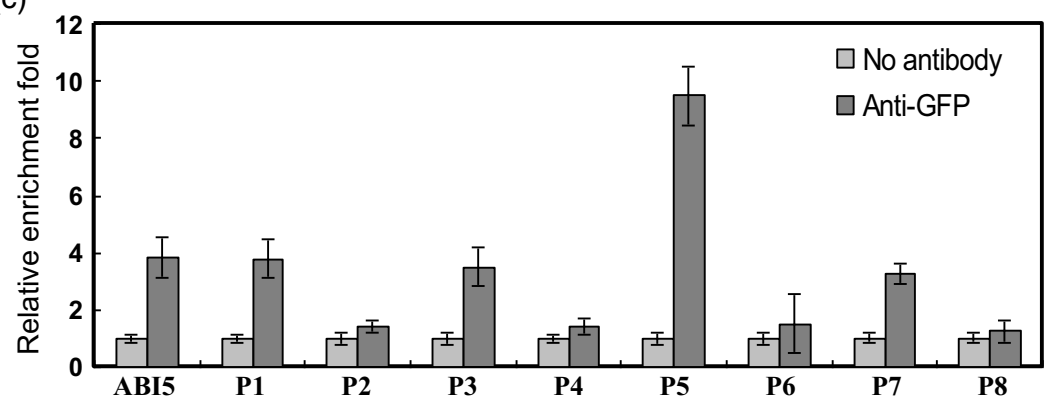

(d)

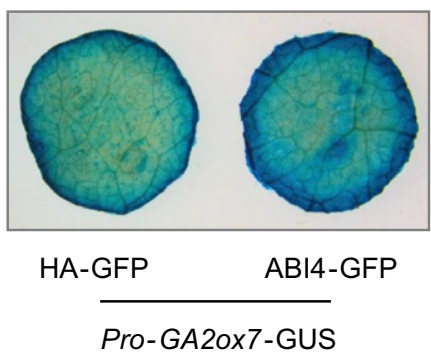

(e)

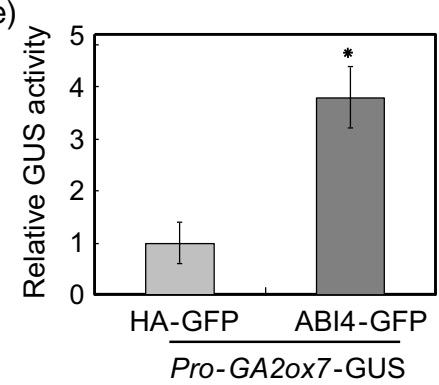

motifs (11 for NCED3 promoter and 8 for $A B A 3$ promoter, respectively).

Taken together, the results presented in Figures $2(b, f)$ and 4 (c) suggest that $A B I 4$ might promote GA2ox7 and NCED6 expression by directly binding to their promoters. To assess this hypothesis, we employed a transient expression system in tobacco (Liu et al., 2010). Two reporter plasmids (Pro-GA2ox7-GUS, Pro-NCED6-GUS) were separately transformed in Nicotiana benthamiana leaves with or without the pCanG-ABI4-GFP or pCanG$H A-G F P$ (negative control) effector plasmids, and GUS levels were detected by a qualitative (Figure 4d) and a quantitative (Figure 4e) assay. Leaves co-transformed with the pCanG-ABI4-GFP and Pro-GA2ox7-GUS constructs showed a significant increase in GUS levels in both assays when compared with leaves co-transformed with the pCanG-HA-GFP and Pro-GA2ox7-GUS constructs. Similar effects of $A B I 4$ on the expression of NCED6 were also detected using the same system (Figure S7a,b). These results confirm that $\mathrm{ABI} 4$ has a direct effect on GA2ox7 and NCED6 transcription in vivo.

\section{GA2ox7 and NCED6 mutations partially rescue the dwarf phenotype of $O E-A B I 4$}

Our finding that $O E-A B / 4$ transgenic plants have a decreased $G A / A B A$ ratio suggests that their phenotypic defects might be related to this hormonal imbalance. To confirm this hypothesis, we genetically dissected the relationship between GA20x7, NCED6 and $A B / 4$.

To this end, we introduced the 35 S-ABI4-GFP construct into the knock-out mutants ga2ox7 (SALK_055721C) and nced6 (CS852600) (Magome et al., 2008; Toh et al., 2008) and subjected them to phenotypic analysis. As shown in Figure 5(a,b), neither ga2ox7 nor nced6 mutant seedlings have a noticeable phenotype when compared with the WT. However, the ga2ox7 mutation partially rescued the dwarf phenotype of adult $O E-A B I 4$ plants (Figure $5 \mathrm{a}$, top panel). Similarly, the nced6 mutation partially restored the WT phenotype of $O E-A B / 4$ transgenic plants (Figure 5b, top panel). This partial phenotypic rescue does not seem to be due to altered expression of the transgene, since the fusion protein is expressed at comparable levels in all lines exam- 
(a)
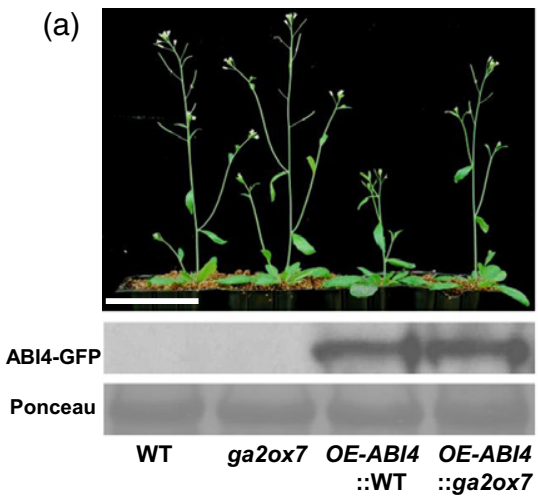

(c)

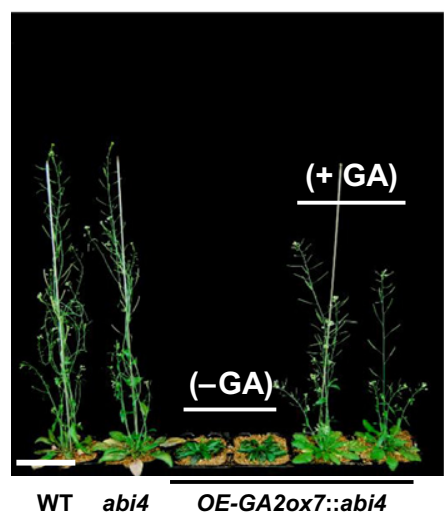

(b)

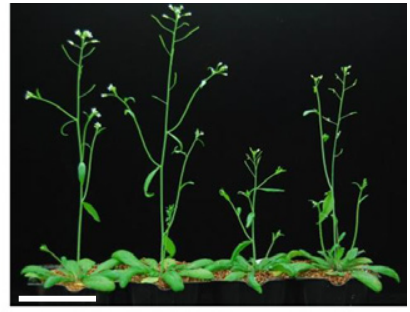

AB14-GFP

Ponceau

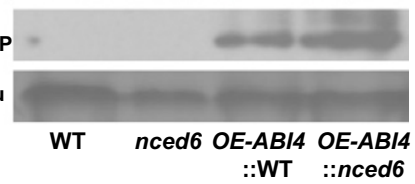

(d)

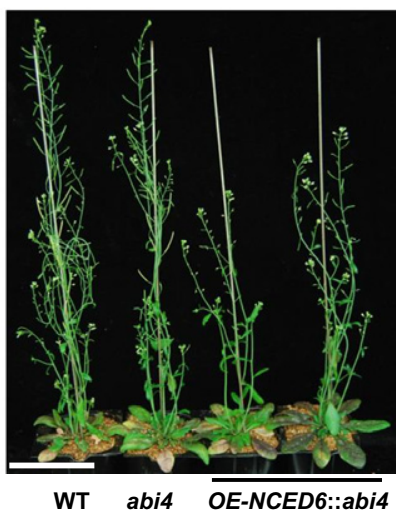

Figure 5. $A B / 4$ acts genetically upstream of $G A 20 x 7$ and NCED6.

(a) Phenotypic comparison (top panel) and relative immunoblot analysis (bottom panel) of 35-day-old plants from WT (wild type), ga2ox7, OE-AB/4 and $O E-A B / 4$ in a ga2ox7 background $(O E-A B / 4:$ : ga2ox7).

(b) Phenotypic comparison (top panel) and relative immunoblot analysis (bottom panel) of 35-day-old plants from WT, nced6, $O E-A B / 4$, and $O E-A B / 4$ in a nced6 background (OE-ABI4::nced6).

(c) Phenotypic comparison of WT, abi4 and OE-GA2ox7::abi4 plants (7 weeks old), grown with or without $100 \mu \mathrm{m} \mathrm{GA}$.

(d) Phenotypic comparison of WT, abi4 and OE-NCED6::abi4 plants (7 weeks old). Bar $=20 \mathrm{~mm}$ ined (Figure $5 a, b$, lower panels). Taken together, these genetic analyses indicate that the dwarf stature of $O E-A B / 4$ plants is partially dependent on the ABI4-induced expression of GA2ox7 and NCED6 and suggest that GA2ox7 and NCED6 may act genetically downstream of $A B I 4$.

To further evaluate this hypothesis, we analyzed the phenotype of abi4 mutant plants overexpressing GA2ox7. As shown in Figure 5(c), ectopic GA2ox7 expression in the abi4 background greatly reduced plant height (Figure $5 \mathrm{c}$ ). This phenotype mimics the phenotypes of GA2ox7 overexpressing plants or of the ga1-3 mutant (Lee et al., 2002) (Porri et al., 2012). Indeed, similar to ga1-3 and to WT plants overexpressing GA2ox7 (OE-GA2ox7::WT), the $O E$ GA2ox7::abi4 transgenic plants could not bolt unless treated with exogenous GA (Figure $5 c$ ). Similar phenotypes were also detected in abi4 plants overexpressing NCED6 (Figure $5 \mathrm{~d}$ ). These experiments support the hypothesis that GA2ox7 and NCED6 act genetically downstream of $A B / 4$.

\section{The dwarf phenotype of $O E-A B I 4$ is caused by $A B I 4$ rather} than by $A B I 5$

It has been shown that $A B \mid 4$ directly promotes the transcription of $A B I 5$ (ABA- INSENSITIVE 5), another central factor in the $A B A$ signaling pathway (Bossi et al., 2009). Indeed, we have already shown that the $A B I 5$ expression level increased significantly in $O E-A B / 4$ transgenic plants (Shu et al., 2013). To exclude the possibility that the observed dwarf phenotype of $O E-A B \mid 4$ is a result of $A B \mid 4$ induced ectopic expression of $A B I 5$, we overexpressed $A B I 4$ in the abi5-7 mutant background, which has no obvious phenotype under normal growth conditions, compared with its relative WT (Col-0; Figure S8a, and Chen et al., 2012). Among the resulting OE-ABI4::abi5-7 transgenic plants, two independent lines were confirmed to express high levels of $A B / 4$ by qRT-PCR analysis and were thus selected for phenotypic analysis (Figure S8b). As shown in Figure 6(a-c), overexpression of $A B / 4$ in the abi5-7 mutant background also resulted in a dwarf stature in both the seedling (Figure 6a,b) and the adult stage (Figure 6c; compare this with Figure 1d). This evidence demonstrates that the dwarf phenotype of $O E-A B / 4$ transgenic plants is directly caused by $A B I 4$, rather than by $A B I 4 A B I 5$.

\section{ABA and GA have opposite effects on $A B \mid 4$ expression and protein stability}

Our data demonstrate that $A B I 4$ enhances $A B A$ biogenesis while suppressing GA biogenesis (Figures 2 and 3), and suggest that $A B \mid 4$ might regulate $G A / A B A$ homeostasis. We thus speculated that GA and $A B A$ might in turn control $A B I 4$ itself. To assess this hypothesis, we asked whether $\mathrm{GA}$ and $\mathrm{ABA}$ affect $A B / 4$ transcription and protein stability.

To this end, we first monitored the effects of $A B A$ and GA on $A B / 4, G A 20 x 7$ and NCED6 transcription over time. As shown in Figure 7, we found that $A B / 4$ transcription is 
Figure 6. The dwarf phenotype of $O E-A B / 4$ is caused by $A B I 4$ rather than by $A B I 5$.

Post-germination phenotypes of wild-type (WT) and $O E-A B / 4:: a b i 5-7$ plants at different developmental stages.

(a) Sixteen-day-old seedlings, bar $=10 \mathrm{~mm}$.

(b) Eighteen-day-old seedlings, bar $=20 \mathrm{~mm}$.

(c) Forty-day-old plants, bar $=50 \mathrm{~mm}$.

$\# 1$ and \#2 represent two independent $O E-A B / 4::$ abi5-7 transgenic lines.

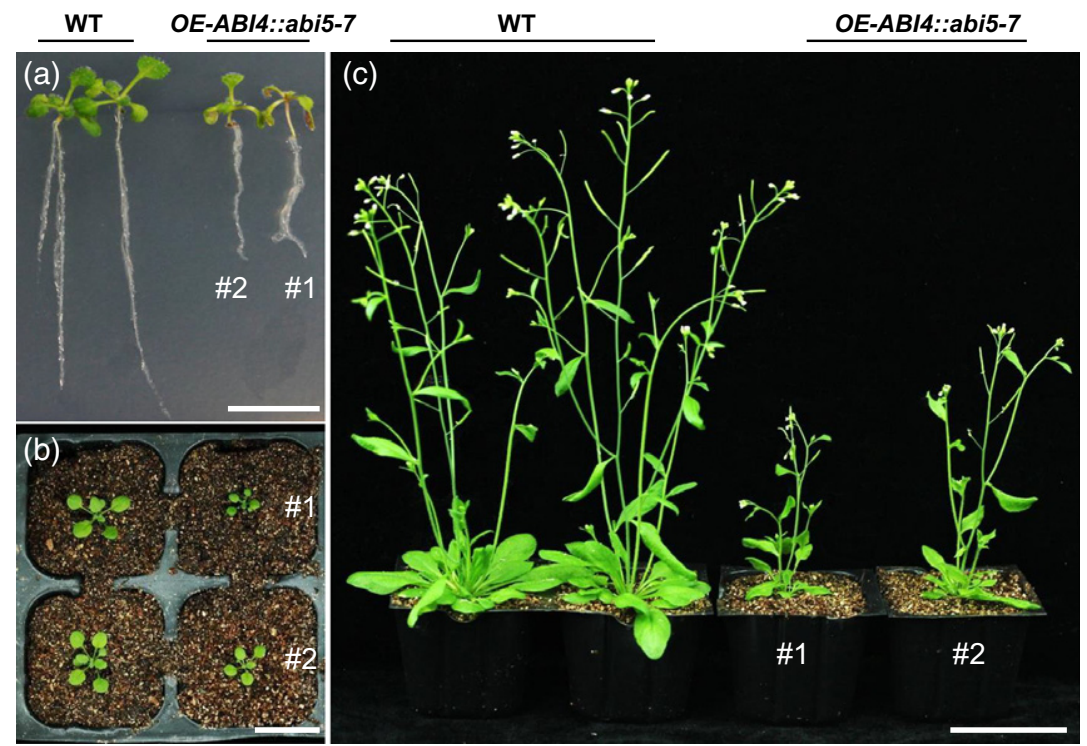

rapidly induced by $A B A$ and suppressed by GA (Figure $7 a$, b). Abscisic acid also significantly induces GA2ox7 expression (Figure 7c) while GA represses NCED6 transcription (Figure 7d). In addition, both ABA-mediated induction of GA20x7 and GA-mediated inhibition of NCED6 require $A B \mid 4$. In detail, both transcriptional induction and inhibition were significantly decreased in the abi4 mutant compared with the WT (Figure 7c-f). Similar results were also obtained when we employed a second reference gene in the qRT-PCR analysis (ACTIN; Figure S9). Because ABI4 directly activates the transcription of GA20x7 and NCED6 (Figures 2 and 4), it is possible that $A B \mid 4$ might partially mediate a cascade amplification effect of $A B A$ and $G A$ through the transcription of these two genes.

As a previous study has demonstrated that the level of $\mathrm{ABI} 4$ protein is regulated - at least partially - by the ubiquitin 26S proteasome system (UPS) (Finkelstein et al., 2011), we next monitored the effects of $A B A$ and $G A$ on the stability of $\mathrm{ABI} 4$ protein over time by incubating seedlings in a medium containing GA or ABA and cycloheximide $(\mathrm{CHX}$; an inhibitor of protein synthesis). As shown in Figure 7(g, h), $\mathrm{ABI} 4$ protein was already degraded after incubation for $30 \mathrm{~min}$ in the presence of GA. On the contrary, ABA was able to induce stabilization of $\mathrm{ABI} 4$ over an incubation time of 45 minutes, while the level of a control protein (Myc-GFP) remained stable over the same time frame (Figure S10). These results conclusively indicate that $A B A$ stabilizes $A B I 4$ while GA enhances its degradation.

\section{DISCUSSION}

Abscisic acid and GA are well known to antagonistically regulate diverse plant growth and development processes (Gale and Marshall, 1973; Ho et al., 1981; Schomburg et al., 2003; Porri et al., 2012). The negative effect of
$A B A$ and the positive effect of $G A$ on plant growth and development are well described in the literature (Nambara and Marion-Poll, 2005; Yamaguchi, 2008; Cutler et al., 2010; Porri et al., 2012). However, the factors regulating the balance between $A B A$ and $G A$, as well as the molecular mechanism of their antagonism, are still not completely clear. Our present study reveals that $A B I 4$ could be part of this mechanism, since it promotes $A B A$ biosynthesis while inhibiting GA biosynthesis by directly regulating the transcription of specific hormone metabolism genes. Further, our study shows that $A B I 4$ also is a key factor that mediates GA/ABA antagonism, since $A B A$ and GA have opposite effects on $A B / 4$ transcription and protein stability.

\section{ABI4 promotes $A B A$ biosynthesis and represses $G A$ biosynthesis}

$A B \mid 4$ has mainly been described as a positive regulator of the ABA signaling pathway (Finkelstein, 1994; Finkelstein et al., 1998; Soderman et al., 2000). $A B / 4$ is highly expressed in maturing and germinating seeds as well as in early seedlings, while it is expressed at relatively low levels during vegetative growth (Soderman et al., 2000). Consistent with this expression pattern, abi4 mutant seedlings develop normally (Soderman et al., 2000), thus preventing a more detailed characterization of the function of ABI4.

To circumvent this problem, we have employed a gainof-function approach to dissect the role of $A B I 4$ in plant growth and development. Similarly to the $A B / 4$ overexpressing plants described by Soderman et al. (2000), our $A B I 4$ overexpressing plants have decresed height, lower seed production and other developmental defects (Figures 1 and $\mathrm{S} 1$ ). $O E-A B / 4$ seedlings also have a decreased $\mathrm{GA} / \mathrm{ABA}$ ratio, which suggests that this imbalance might 


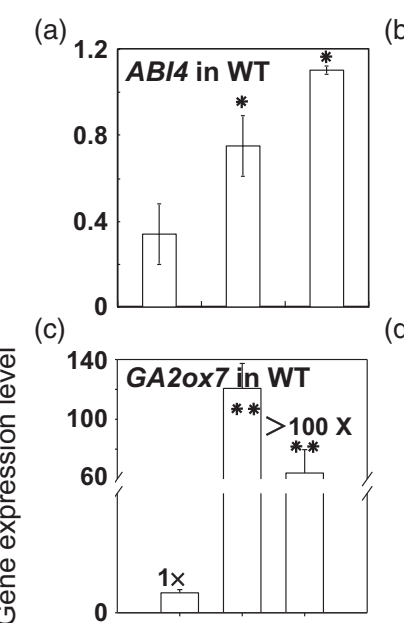

(b)

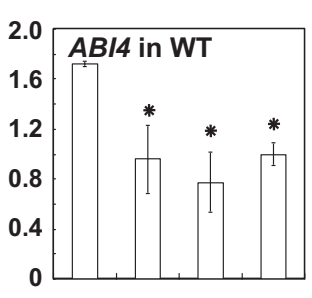

(d)

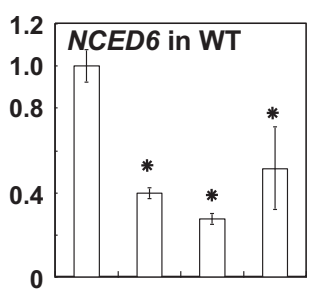

(e)

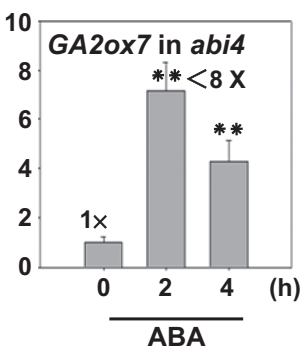

(f)

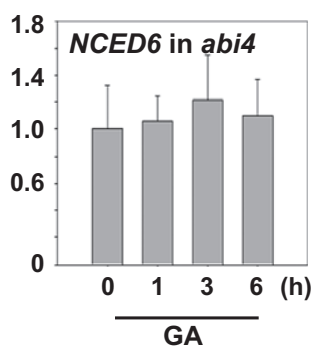

(g)

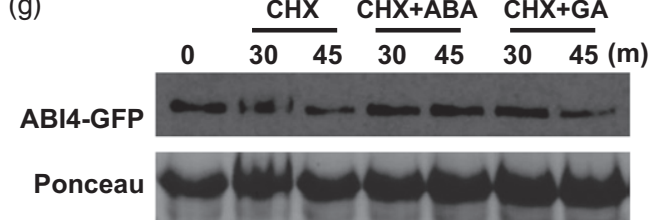

(h)

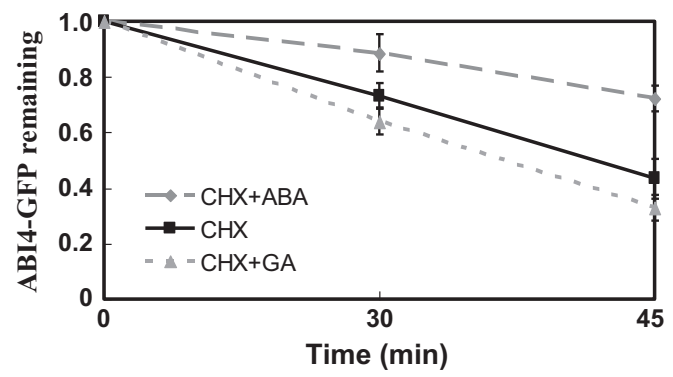

Figure 7. Opposite effect of $A B A$ and gibberellin (GA) on $A B / 4$ transcription and protein stability.

(a)-(f) Effect of $A B A$ and GA on $A B / 4$ transcription. Quantitative RT-PCR analysis of $A B I 4(\mathrm{a}, \mathrm{b}), G A 20 x 7(\mathrm{c}, \mathrm{e})$, and NCED6 (d, f) transcript levels in 2week-old wild type (WT) or abi4 seedlings. Seedlings were treated with ABA $(a, c, e)$ or GA (b, d, f), at the concentrations indicated. Asterisks indicate statistically significant differences from the beginning of the experiment (0) $(* P<0.05 ; * * P<0.01)$. (g) Effect of $A B A$ and $\mathrm{GA}$ on $\mathrm{ABI} 4$ protein levels. Immunoblot analysis (top panel) of 2-week-old homozygous 35S-ABI4-GFP seedlings treated with $50 \mu \mathrm{M} A B A$ or $50 \mu \mathrm{M}$ GA plus or minus $80 \mu \mathrm{M} \mathrm{CHX}$. Antibodies to GFP were used to detect the ABI4-GFP fusion protein. The Ponceau staining (bottom panel) indicates the loading control. The experiment was run in three biological replicates and a typical result is shown. (h) Densitometric analysis of ABI4-GFP degradation shown in (g). The detailed protocol for quantification and normalization is described in the Experimental Procedures. Data are the mean from three biological replicates. Error bars represent SD. be responsible for the phenotypic defects observed in $O E-A B I 4$ transgenic plants.

Previous studies have demonstrated that $A B I 4$, in addition to $A B A$ signaling, is also involved in other pathways, including glucose, cytokinin and JA signaling (Finkelstein, 1994; Finkelstein et al., 1998; Arenas-Huertero et al., 2000; Laby et al., 2000; Soderman et al., 2000; Shkolnik-Inbar and Bar-Zvi, 2010; Kerchev et al., 2011). Here, we further show that $A B I 4$ regulates $A B A$ and GA biogenesis in seedlings (Figure 3). Other AP2 family members seem to share similar functions; Yaish and colleagues have recently demonstrated that - similar to ABI4 - another AP2-like transcription factor, OsAP2-39 from rice (Oryza sativa L.), also enhances $A B A$ biosynthesis and suppresses GA biosynthesis, leading to a decrease in biomass and seed yield (Yaish et al., 2010). A second AP2 family member, DDF1, is a positive regulator of GA20x7 expression, and, when overexpressed, leads to lower GA levels and dwarfism (Magome et al., 2008). Finally, CHOTTO1, a putative double AP2 repeat transcription factor, represses GA biosynthesis during seed germination (Yano et al., 2009).

Combined with these studies, our results suggest that at least some AP2 family members possess specific yet little characterized roles in ABA and GA biogenesis. Recently, by analyzing the phylogenetic history of $A B I 4$ homologs from published proteomes and genomes (a total of 33 species from Phytozome or Plaza, including Arabidopsis thaliana, Thellungiella halophila, Zea mays and Glycine max), a new 'ABI4 motif' (LRPLLPRP) was found, which is conserved across angiosperms (Wind et al., 2013). This remarkable conservation might reflect a role for this domain in mediating the biological functions of $A B I 4$. Further experiments will be required to assess this hypothesis.

\section{$A B I 4$ oppositely regulates the transcription of $A B A$ and $G A$ metabolism genes}

Our data indicate that $A B I 4$ regulates the levels of $A B A$ and GA by binding to the promoters of the NCED6 and GA20x7 genes and promoting their transcription (Figures 4 and S7). This conclusion is further supported by the partial rescue of the $O E-A B \mid 4$ dwarf phenotype by the ga2ox7 and nced6 mutations (Figure 5).

GA20x7 overexpression has been shown to significantly reduce the levels of bioactive $\mathrm{GA}$, resulting in a dwarf phenotype in Arabidopsis and tobacco (N. tabacum) (Schomburg et al., 2003; Magome et al., 2008; Tong et al., 2009; Porri et al., 2012). Furthermore, constitutive overexpression of NCED1 in tomato (Solanum lycopersicum) and of NCED2, NCED3 and NCED5 in Arabidopsis increased the $A B A$ content in transgenic plants and caused similar phenotypes (Thompson et al., 2000; Fan et al., 2009). NCED6 overexpressing transgenic plants also showed a reduced rosette size due to an increase in the level of ABA (Lefebvre 
et al., 2006). In agreement with these studies, we show that GA20x7 and NCED6 are upregulated in OE-AB/4 plants, and consequently that the GA and ABA contents are altered in the opposite manner (Figures 2 and 3). We also provide molecular and genetic evidence that GA20x7 and NCED 6 are direct targets of $\mathrm{ABI} 4$ (Figures 4, 5 and S7). Taken together, our results demonstrate that $A B I 4$ mediates $\mathrm{GA} / \mathrm{ABA}$ homeostasis by directly regulating the transcription of specific genes involved in the GA and $A B A$ metabolism pathways.

\section{$A B A$ and $G A$ antagonize each other by oppositely affecting $A B / 4$ transcription and protein stability}

The antagonistic relationship between $G A$ and $A B A$ has been extensively studied (Rymen and Sugimoto, 2012; Vanstraelen and Benkova, 2012). However, the detailed molecular mechanisms underlying the antagonistic effect between these two hormones are still largely unknown.

A possible target of $A B A$ and $G A$ is miR159, which regulates the expression of the MYB33 gene (Achard et al., 2004; Reyes and Chua, 2007). However, both ABA and GA induce accumulation of miR159 - and consequently downregulation of $M Y B 33$ - thus promoting $A B A$ responses in seeds and GA responses in flowers (Gocal et al., 2001; Reyes and Chua, 2007). This implies that MYB33 is not a likely mediator of $A B A / G A$ antagonism, but rather a common effector of the two hormones in two different developmental processes. On the contrary, in our present study we demonstrate that $A B A$ and $G A$ exert opposite effects on $A B / 4$ transcript and protein levels; $A B A$ induces $A B I 4$ transcription while GA inhibits it (Figure 7a,b): $A B \mid 4$ is stabilized by $A B A$, while GA promotes its degradation (Figure $7 \mathrm{~g}, \mathrm{~h}$ ).

We also show that $A B A$ induces transcription of GA20x7 while GA inhibits expression of NCED6 in an ABI4-dependent manner (Figure 7c-f). Based on these results, we propose the working model shown in Figure 8. Briefly, $A B A$ induces transcription of $A B / 4$ and protein stabilization; the resulting higher level of $A B \mid 4$ promotes expression of GA2ox7, thus downregulating GA biogenesis. In contrast, $\mathrm{GA}$ inhibits the expression of $A B / 4$ and enhances degradation of its protein, thus inhibiting transcription of NCED6 and impairing $A B A$ biosynthesis. Therefore, $A B A$ and $G A$ antagonize each other by altering both transcription of $A B I 4$ and its protein levels, and, consequently, transcription of GA2ox7 and NCED6. ABI4 could possibly represent a key target of GA/ABA antagonism, and provide one of the missing links between $A B A$ production and GA inactivation.

\section{ABI4 transcript and protein levels are tightly regulated}

Our working model shown in Figure 8 depicts positive feedback between $A B A$ biogenesis, $A B / 4$ transcription and the level of $A B I 4$ protein. On the other hand, the

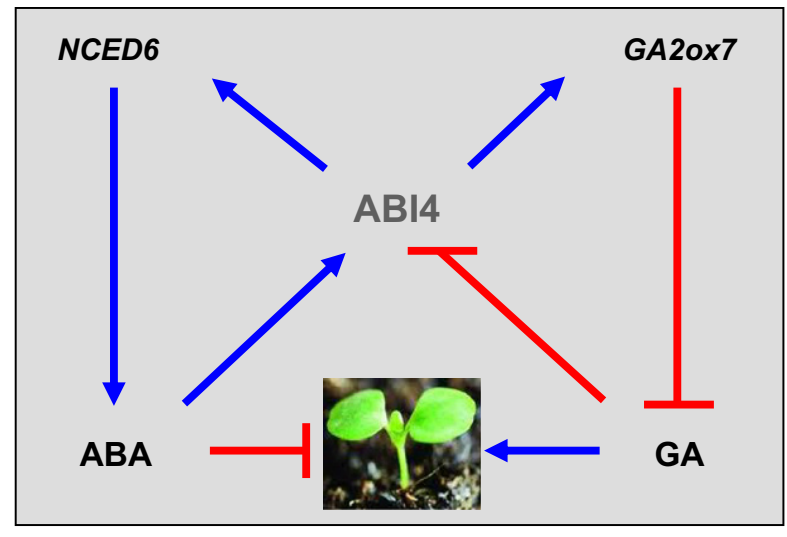

Figure 8. Proposed working model of the antagonistic effects of gibberellin (GA)/ABA on $\mathrm{ABI}$.

$A B I 4$ promotes $A B A$ biosynthesis and repress $G A$ biogenesis by the direct activation of NCED6 and GA2ox7, and therefore negatively regulates plant growth and development. Conversely, $A B A$ induces GA2ox7 expression and GA inhibits NCED6 transcription in an ABI4-dependent manner. In addition, $A B A$ maintains a proper level of $A B I 4$ protein, while GA promotes degradation of $A B \mid 4$. This model proposes that $A B A$ antagonizes $G A$ by promoting $A B / 4$ transcription and protein stabilization, thus enhancing transcription of GA20x7 and eventually decreasing GA biogenesis. Vice versa, GA antagonizes $A B A$ by inhibiting $A B / 4$ expression and enhancing degradation of its protein, thus, attenuating expression of NCED 6 and impairing biogenesis of $A B A$. Blue lines ending with arrows denote positive regulation, while red lines ending with bars denote negative regulation.

model predicts that higher ABA levels further inhibit GA biosynthesis through activation of GA20x7 expression, thus explaining the dwarf stature of $A B / 4$ overexpression lines (Figures 1 and 8). Because optimal hormone levels are essential for normal plant development, the $A B A$ and GA-mediated regulation of $\mathrm{ABI} 4$ might represent $a$ key mechanism for finely regulating the plant life cycle. However, the molecular mechanism through which ABA and $\mathrm{GA}$ regulate levels of $\mathrm{ABI} 4$ transcript and protein still requires further characterization. Recently, members of the PTM (PHD-type transcription factor with transmembrane domains) transcription factor family were identified as activators of $A B / 4$ expression (Sun et al., 2011). Furthermore, it has been shown that $\mathrm{ABI} 4$ also promotes its own transcription by binding directly to its promoter (Bossi et al., 2009). On the other hand, repressors of $A B / 4$ expression have been recently found: the transcription factors WRKY40, WRKY18 and WRKY60 bind to the $A B / 4$ promoter and suppress its expression (Shang et al., 2010; Liu et al., 2012). However, although these WRKY transcription factors directly inhibit expression of $A B I 4$, introduction of the abi4 mutation into the wrky18 and wrky60 mutants led to double mutants with an abi4 phenotype on ABA-containing medium (Liu et al., 2012). These results demonstrate that $A B / 4$ is a target of these two WRKY transcription factors, but do not give full insight into the molecular mode of action of $A B I 4$. 
As for the ABI4 protein level, a previous study has already shown that $A B I 4$ degradation is at least partially mediated by the UPS (Finkelstein et al., 2011). In line with this result, our present study shows that ABI4 is degraded via the UPS: however, the specific E3 ubiquitin ligase(s) responsible for $\mathrm{ABI} 4$ ubiquitination have not yet been found. Therefore, the regulatory mechanism through which $G A$ and $A B A$ precisely regulate the optimal levels of ABI4 mRNA and protein still awaits further investigation. Clearly, a genetic screen for suppressors of the abi4 mutant phenotype could represent a worthwhile future study.

\section{EXPERIMENTAL PROCEDURES}

\section{Plant materials and growth conditions}

The Arabidopsis ecotype Col-0 was used as the WT in this study. The point mutant abi4-1 (CS8104) and the ga20x7 (SALK_055721C) and nced6 (CS852600) mutants were ordered from the Arabidopsis Biological Resource Center (ABRC; https:// abrc.osu.edu/). The abi5-7 mutant is of the Col-0 ecotype and was kindly provided by $\mathrm{Dr}$ Chuanyou Li, Institute of Genetics and Developmental Biology, Chinese Academy of Sciences. Arabidopsis seeds were surface-sterilized with $10 \%$ bleach and washed at least four times with sterile water. Seeds were suspended in $0.2 \%$ agarose and sowed on $1 / 2$ MS solid medium plus $1 \%$ sucrose. Plates were stratified under darkness for 3 days in a $4^{\circ} \mathrm{C}$ cold room and then transferred to the tissue culture room at $22^{\circ} \mathrm{C}$ under a 16 -h light/8-h dark photoperiod. After about 2 weeks, seedlings on $1 / 2$ MS were potted in soil and placed in a growth chamber (16-h light/8-h dark photoperiod) at $22^{\circ} \mathrm{C}$ and $70 \%$ relative humidity condition. The $1 / 2 \mathrm{MS}$ medium was supplemented with PAC (product number 46046, Sigma-Aldrich, http://www.sigmaaldrich.com// as indicated in the text. For spraying the plants, $100 \mu \mathrm{m} \mathrm{GA}$ was used.

\section{Constructs and transgenic plants}

Transgenic plants constitutively expressing $A B I 4$ in the WT background were described in Shu et al. (2013). Transgenic plants overexpressing ABI4-GFP (35S-ABI4-GFP) (Shu et al., 2013), GA2ox7-GFP (OE-GA2ox7-GFP) or NCED6-GFP (OE-NCED6-GFP) in the WT or in the abi5-7 background were generated by PCR amplification of the coding sequences of $A B / 4, G A 20 \times 7$ and NCED6, followed by cloning into the binary vector pCanG-HAGFP under the control of the CaMV 35 S promoter. Transformation of Arabidopsis was conducted by the vacuum infiltration method using Agrobacterium tumefaciens strain EHA105 (Bechtold and Pelletier, 1998). $\mathrm{T}_{2}$ seeds were germinated on MS plates containing $50 \mathrm{mg} \mathrm{ml}^{-1}$ kanamycin, and the resistant seedlings were transferred to soil to obtain homozygous $T_{3}$ seeds. The expression levels of the transgenes were determined by qRT-PCR analyses. Independent $\mathrm{T}_{3}$ homozygous lines containing a single insertion were employed in the subsequent phenotypic and physiological analyses.

To generate ga2ox7 and nced 6 plants overexpressing ABI4-GFP, the 35S-ABI4-GFP construct was crossed into the ga2ox7 and the nced 6 background, respectively. The $\mathrm{F}_{2}$ progenies were tested by PCR using the specified primers for ga2ox7 and nced6 genotyping. The ABI4-GFP protein level was monitored through immunoblot analysis for all lines, and the lines which possessed a comparable ABI4-GFP protein level were selected and used for further genetic analysis.

\section{Gene expression analyses}

Total RNA preparation from about 2-week-old seedlings, firststrand cDNA synthesis and qRT-PCR were performed following a previous protocol (Cui et al., 2012). DNasel-treated total RNA $(2 \mu \mathrm{g})$ was denatured and subjected to reverse transcription using Moloney murine leukemia virus reverse transcriptase (200 units per reaction; Promega, http://www.promega.com/). Quantitative RT-PCR was performed using the SsoFast ${ }^{\mathrm{TM}}$ EvaGreen Supermix (Bio-Rad, http://www.bio-rad.com/) and CFX96 Touch ${ }^{\text {TM }}$ Real-Time PCR Detection System (Bio-Rad). Gene expression was quantified at the logarithmic phase using expression of the housekeeping $18 S$ RNA as an internal control.

\section{Genetic and immunoblot analyses}

For total protein extracts, 2-week-old seedlings were ground in liquid nitrogen and extracted with $4 \mathrm{~m}$ urea buffer. Crude extracts were separated by SDS-PAGE and transferred to nitrocellulose membranes. The membranes were stained with $0.2 \%$ Ponceau $S$, with Rubisco functioning as an internal control. The antibody to GFP was purchased from Santa Cruz Biotechnology, Inc. (http:// www.scbt.com/). For the experiment on the effect of $A B A$ and $G A$ on $\mathrm{ABI} 4$ protein stability, 2-week-old homozygous 35 S-ABI4-GFP seedlings were treated with $50 \mu \mathrm{m}$ ABA plus $80 \mu \mathrm{m} \mathrm{CHX,} \mathrm{or} 50 \mu \mathrm{m}$ GA plus $80 \mu \mathrm{m} \mathrm{CHX,} \mathrm{for} \mathrm{the} \mathrm{time} \mathrm{indicated} \mathrm{in} \mathrm{the} \mathrm{text.}$

\section{Protein quantification and normalization}

To quantify and normalize the amount of protein in the protein degradation assay, we employed the standard software ImageJ (National Institutes of Health, http://imagej.nih.gov/ij/) according to a previous protocol (Zhang et al., 2011a). The percentage of ABI4-GFP remaining in the degradation assay was normalized by the formula $P_{\mathrm{t}}=\left(C_{\mathrm{t}} / L_{\mathrm{t}}\right) \times 100 \%$, where $P_{\mathrm{t}}$ is the percentage of the test protein remaining in each sampling time point, $C_{\mathrm{t}}$ is the quantified content of the test protein in each sampling time point and $L_{\mathrm{t}}$ is the loading content in each sampling time point. In particular, $P_{0}=\left(C_{0} / L_{0}\right) \times 100 \%$, where $P_{0}$ is the percentage of the test protein remaining at $0 \mathrm{~h}, C_{0}$ is the quantified content of the test protein at $0 \mathrm{~h}$ and $L_{0}$ is the loading content at $0 \mathrm{~h}$. $P_{0}$ was normalized as 1.00 , and the values of different $P_{\mathrm{t}} \mathrm{s}$ were calculated.

\section{Quantification of ABA}

Two-week-old seedlings were ground in liquid nitrogen, and $250 \mathrm{mg}$ frozen powder was homogenized and extracted for $24 \mathrm{~h}$ in methanol containing D6-ABA (purchased from OIChemlm Ltd, http://www.olchemim.cz//) as an internal standard. Purification was performed with an Oasis Max solid phase extract cartridge (150 mg/6 cm $\mathrm{cm}^{3}$; Waters, http://www.waters.com/) and eluted with $5 \%$ formic acid in methanol. The elution was dried, reconstituted and finally injected into a liquid chromatography-tandem mass spectrometry system consisting of an Acquity ultra performance liquid chromatograph (Acquity UPLC; Waters) and a triple quadrupole tandem mass spectrometer (Quattro Premier XE; Waters). Three biological replicates were performed.

\section{Quantification of GA}

The endogenous GA content was determined in the Key Laboratory of Analytical Chemistry for Biology and Medicine (Ministry of Education), Wuhan University, China, by a previous method (Chen 
et al., 2011). Briefly, Arabidopsis seedlings (100 mg) were frozen in liquid nitrogen, ground to a fine powder and extracted with $80 \%(\mathrm{v} / \mathrm{v})$ methanol. Before grinding GA isotope standards were added to the plant samples. The crude extracts were purified by reverse-phase solid-phase extraction, ethyl ether extraction and derivatization. The resulting mixture was injected into a capillary electrophoresis-mass spectrometer (CE-MS) for quantitative analysis.

\section{Chromatin immunoprecipitation-qPCR assay}

Chromatin Immunoprecipitation was performed as previously described with minor modifications (Lu et al., 2011). 35S-ABI4GFP transgenic seedlings grown on $1 / 2$ MS plates for about 2 weeks were sampled $(1.5 \mathrm{~g})$ and cross-linked by $1 \%$ formaldehyde for $30 \mathrm{~min}$ in a vacuum, and stopped by $0.125 \mathrm{~m}$ glycine. Seedlings were ground in liquid nitrogen and nuclei were isolated. Immunoprecipitations were performed with anti-GFP antibody and protein $\mathrm{G}$ beads. DNA was precipitated by isopropanol, washed by $70 \%$ ethanol and then dissolved in $10 \mu \mathrm{l}$ water within $20 \mu \mathrm{g} \mathrm{ml}^{-1}$ RNase. Absence of anti-GFP functions as a control. Quantitative PCR analysis was performed using specific primers corresponding to different promoter regions of GA20x7 and NCED6. TUB4 was used as an internal control. Because it is known that $A B I 4$ binds directly to the promoter of $A B I 5$ (Bossi et al., 2009), we employed the $A B I 5$ promoter as a positive control.

\section{In vivo transient analysis of GA20x7 and NCED6 promoter activity by $\mathrm{ABI} 4$}

Native GA20x7 and NCED6 promoters (Pro- GA20x7 and ProNCED6) were amplified by PCR from genomic DNA. Primer sequences are listed in Table S1. Fragments of both promoters were cloned in the $p$ Cambia1300-221 vector by replacing the original CaMV 35S promoter, thus generating the Pro-GA2ox7-GUS and Pro-NCED6-GUS constructs. The effector construct pCanGABI4-GFP was generated in our previous study (Shu et al., 2013). Agrobacterium tumefaciens-mediated tobacco transient transformation was performed according to our previous protocol (Liu et al., 2010). Briefly, Agrobacterium cells containing the appropriate construct combinations were cultured at $28^{\circ} \mathrm{C}$ overnight, collected, re-suspended with infiltration buffer and infiltrated into healthy tobacco ( $N$. benthamiana) leaves. Total proteins were extracted from the infiltrated leaves and GUS activity was determined using the protocol described previously using 4-methylumbelliferyl- $\beta$-D-glucuronide (Sigma-Aldrich) as a substrate (Jefferson et al., 1987). The total protein was quantified using the Bradford protein assay kit method (Bio-Rad). At the same time, histochemical staining for GUS was performed on leaves at 3 days after infiltration using a hole punch according to a protocol published elsewhere (Stalberg et al., 1993). Photographs were taken using a Leica MZ16 FA stereomicroscope (Leica Company, http://www.leica.com/).

\section{ACCESSION NUMBERS}

Arabidopsis Genome Initiative locus identifiers for the major genes mentioned in this article are as follows: $A B / 4$ (AT2G40220), ABI5 (AT2G36270), GA20x7 (AT1G50960), NCED6 (AT3G24220), GA20ox1 (AT4G25420), GA20ox2 (AT5G51810), AtEXP2 (AT5G05290), NCED3 (AT3G14440), ABA3 (AT1G16540), RGL2 (AT3G03450), RGL3 (AT5G17490), DDF1 (AT1G12610), CYP707A2 (AT2G29090) and CYP707A3
(AT5G45340). The stock numbers of the mutants used in this study are as follows: abi4 (CS8104), ga2ox7 (SALK_055721C) and nced6 (WiscDsLox356H02, also named CS852600).

\section{ACKNOWNLEDGEMENTS}

We thank Drs Jinfang Chu, Cunyu Yan and Xiaohong Sun (National Centre for Plant Gene Research (Beijing), Institute of Genetics and Developmental Biology, Chinese Academy of Sciences, Beijing) for determining the ABA content. We also thank Drs Yuqi Feng and Mingluan Chen, Department of Chemistry, Wuhan University, for active GA measurement. The T-DNA insertion mutants were provided by the Arabidopsis Biological Resource Center (ABRC). abi5-7 mutant seeds (Col-0 background) were kindly provided by Dr Chuanyou Li (Institute of Genetics and Developmental Biology, Chinese Academy of Sciences). This research was supported by the grants NSFC 91317308 from the National Science Foundation of China, National Basic Research Program of China (2012CB114300) and China Postdoctoral Science Foundation funded project (2014M552377) to KS.

\section{SUPPORTING INFORMATION}

Additional Supporting Information may be found in the online version of this article.

Figure S1. Quantification of selected phenotypes of wild-type, abi4 and $O E-A B I 4$ plants at different developmental stages.

Figure S2. Size analysis of wild-type, abi4 and $O E-A B / 4$ seeds.

Figure S3. Effects of $A B I 4$ loss or overproduction on selected gibberellin and $A B A$ biogenesis genes.

Figure S4. ABI4 regulates the transcription profiles of specific gibberellin and $A B A$ biogenesis genes.

Figure S5. Effect of exogenous ABA on CYP707A2 and CYP707A3 transcription.

Figure S6. $A B I 4$ does not affect DDF1 transcription.

Figure S7. ABI4 activates NCED6 transcription in vivo.

Figure S8. Confirmation of $O E-A B I 4:: a b i 5-7$ transgenic lines by quantitative PCR.

Figure S9. Opposite effect of $A B A$ and gibberellin on $A B / 4$ transcription.

Figure S10. Exogenous $A B A$ and gibberellin have no effect on the stability of Myc-GFP protein.

Table S1. Primers used in this study.

\section{REFERENCES}

Acevedo-Hernandez, G.J., Leon, P. and Herrera-Estrella, L.R. (2005) Sugar and $A B A$ responsiveness of a minimal RBCS light-responsive unit is mediated by direct binding of ABI4. Plant J. 43, 506-519.

Achard, P., Herr, A., Baulcombe, D.C. and Harberd, N.P. (2004) Modulation of floral development by a gibberellin-regulated microRNA. Development, 131, 3357-3365.

Arenas-Huertero, F., Arroyo, A., Zhou, L., Sheen, J. and Leon, P. (2000) Analysis of Arabidopsis glucose insensitive mutants, gin 5 and gin6, reveals a central role of the plant hormone $A B A$ in the regulation of plant vegetative development by sugar. Genes Dev. 14, 2085-2096.

Barrero, J.M., Piqueras, P., Gonzalez-Guzman, M., Serrano, R., Rodriguez, P.L., Ponce, M.R. and Micol, J.L. (2005) A mutational analysis of the $A B A 1$ gene of Arabidopsis thaliana highlights the involvement of $A B A$ in vegetative development. J. Exp. Bot. 56, 2071-2083.

Bechtold, N. and Pelletier, G. (1998) In planta Agrobacterium-mediated transformation of adult Arabidopsis thaliana plants by vacuum infiltration. Methods Mol. Biol. 82, 259-266.

Bossi, F., Cordoba, E., Dupre, P., Mendoza, M.S., Roman, C.S. and Leon, P. (2009) The Arabidopsis ABA-INSENSITIVE (ABI) 4 factor acts as a central transcription activator of the expression of its own gene, and for the 
induction of $A B / 5$ and SBE2.2 genes during sugar signaling. Plant J. 59, 359-374.

Busov, V.B., Brunner, A.M. and Strauss, S.H. (2008) Genes for control of plant stature and form. New Phytol. 177, 589-607.

Chen, M.L., Huang, Y.Q., Liu, J.Q., Yuan, B.F. and Feng, Y.Q. (2011) Highly sensitive profiling assay of acidic plant hormones using a novel mass probe by capillary electrophoresis-time of flight-mass spectrometry. J. Chromatogr. B, 879, 938-944.

Chen, R., Jiang, H., Li, L. et al. (2012) The Arabidopsis Mediator subunit MED25 differentially regulates jasmonate and ABA signalings through interacting with MYC2 and ABI5. Plant Cell, 24, 2898-2916.

Cheng, H., Qin, L.J., Lee, S.C., Fu, X.D., Richards, D.E., Cao, D.N., Luo, D. Harberd, N.P. and Peng, J.R. (2004) Gibberellin regulates Arabidopsis floral development via suppression of DELLA protein function. Development, 131, 1055-1064.

Cui, F., Liu, L.J., Zhao, Q.Z., Zhang, Z.H., Li, Q.L., Lin, B.Y., Wu, Y.R., Tang, S.Y. and Xie, 0. (2012) Arabidopsis ubiquitin conjugase UBC32 Is an ERAD component that functions in brassinosteroid-mediated salt stress tolerance. Plant Cell, 24, 233-244.

Curaba, J., Moritz, T., Blervaque, R., Parcy, F., Raz, V., Herzog, M. and Vachon, G. (2004) AtGA3ox2, a key gene responsible for bioactive gib berellin biosynthesis, is regulated during embryogenesis by LEAFY COTYLEDON2 and FUSCA3 in Arabidopsis. Plant Physiol. 136, 3660 3669.

Cutler, S.R., Rodriguez, P.L., Finkelstein, R.R. and Abrams, S.R. (2010) Abscisic acid: emergence of a core signaling network. Annu. Rev. Plant Biol. 61, 651-679.

Fan, J., Hill, L., Crooks, C., Doerner, P. and Lamb, C. (2009) Abscisic acid has a key role in modulating diverse plant-pathogen interactions. Plant Phys iol. 150, 1750-1761.

Feng, S., Martinez, C., Gusmaroli, G. et al. (2008) Coordinated regulation of Arabidopsis thaliana development by light and gibberellins. Nature, $\mathbf{4 5 1}$ 475-479.

Finkelstein, R.R. (1994) Mutations at 2 new Arabidopsis Aba response loci are similar to the Abi3 mutations. Plant J. 5, 765-771.

Finkelstein, R.R., Wang, M.L., Lynch, T.J., Rao, S. and Goodman, H.M (1998) The Arabidopsis abscisic acid response locus ABI4 encodes an APETALA2 domain protein. Plant Cell, 10, 1043-1054.

Finkelstein, R., Lynch, T., Reeves, W., Petitfils, M. and Mostachetti, M. (2011) Accumulation of the transcription factor ABA-insensitive $(\mathrm{ABI}) 4$ is tightly regulated post-transcriptionally. J. Exp. Bot. 62, 3971 3979.

Fujii, H. and Zhu, J.K. (2009) Arabidopsis mutant deficient in 3 abscisic acid activated protein kinases reveals critical roles in growth, reproduction, and stress. Proc. Natl Acad. Sci. USA, 106, 8380-8385.

Gale, M.D. and Marshall, G.A. (1973) Insensitivity to gibberellin in Dwarf wheats. Ann. Bot. 37, 729-735.

Gazzarrini, S., Tsuchiya, Y., Lumba, S., Okamoto, M. and McCourt, P. (2004) The transcription factor FUSCA3 controls developmental timing in Arabidopsis through the hormones gibberellin and abscisic acid. Dev. Cell, 7, 373-385.

Giraud, E., Van Aken, O., Ho, L.H.M. and Whelan, J. (2009) The transcription factor $A B I 4$ is a regulator of mitochondrial retrograde expression of ALTERNATIVE OXIDASE1a. Plant Physiol. 150, 1286-1296.

Gocal, G.F.W., Sheldon, C.C., Gubler, F. et al. (2001) GAMYB-like genes, flowering, and gibberellin signaling in Arabidopsis. Plant Physiol. 127, 1682-1693.

Gray, W.M. (2004) Hormonal regulation of plant growth and development. PLoS Biol. 2, 1270-1273.

Ho, T.H.D., Nolan, R.C. and Shute, D.E. (1981) Characterization of a Gibberellin-Insensitive Dwarf Wheat, D6899 - evidence for a regulatory step common to many diverse responses to Gibberellins. Plant Physiol. 67 1026-1031.

Jefferson, R.A., Kavanagh, T.A. and Bevan, M.W. (1987) Gus fusions - betaglucuronidase as a sensitive and versatile gene fusion marker in higherplants. EMBO J. 6, 3901-3907.

Kerchev, P.I., Pellny, T.K., Vivancos, P.D., Kiddle, G., Hedden, P., Driscoll, S. Vanacker, H., Verrier, P., Hancock, R.D. and Foyer, C.H. (2011) The transcription factor $\mathrm{ABI} 4$ Is required for the ascorbic acid-dependent regulation of growth and regulation of jasmonate-dependent defense signaling pathways in Arabidopsis. Plant Cell, 23, 3319-3334.
Ko, J.H., Yang, S.H. and Han, K.H. (2006) Upregulation of an Arabidopsis RING-H2 gene, XERICO, confers drought tolerance through increased abscisic acid biosynthesis. Plant J. 47, 343-355.

Koussevitzky, S., Nott, A., Mockler, T.C., Hong, F., Sachetto-Martins, G. Surpin, M., Lim, I.J., Mittler, R. and Chory, J. (2007) Signals from chloroplasts converge to regulate nuclear gene expression. Science, $\mathbf{3 1 6}, 715$ 719.

Laby, R.J., Kincaid, M.S., Kim, D.G. and Gibson, S.I. (2000) The Arabidopsis sugar-insensitive mutants sis 4 and sis 5 are defective in abscisic acid synthesis and response. Plant J. 23, 587-596.

Lee, S., Cheng, H., King, K.E., Wang, W., He, Y., Hussain, A., Lo, J., Harberd N.P. and Peng, J. (2002) Gibberellin regulates Arabidopsis seed germination via $\mathrm{RGL2}$, a GAl/RGA-like gene whose expression is up-regulated following imbibition. Genes Dev. 16, 646-658.

Lee, L.Y.C., Hou, X.L., Fang, L., Fan, S.G., Kumar, P.P. and Yu, H. (2012) STUNTED mediates the control of cell proliferation by GA in Arabidop sis. Development, 139, 1568-1576.

Lee, K.P., Piskurewicz, U., Tureckova, V., Strnad, M. and Lopez-Molina L. (2010) A seed coat bedding assay shows that RGL2-dependen release of abscisic acid by the endosperm controls embryo growth in Arabidopsis dormant seeds. Proc. Natl Acad. Sci. USA, 107, 19108 19113.

Lefebvre, V., North, H., Frey, A., Sotta, B., Seo, M., Okamoto, M., Nambara, E. and Marion-Poll, A. (2006) Functional analysis of Arabidopsis NCED6 and NCED9 genes indicates that ABA synthesized in the endosperm is involved in the induction of seed dormancy. Plant J. 45, 309319

Li, P., Zhou, H., Shi, X. et al. (2014) The ABI4-induced Arabidopsis ANAC060 transcription factor attenuates $A B A$ signaling and renders seedlings sugar insensitive when present in the nucleus. PLoS Genet. 10, e1004213.

Liu, L.J., Zhang, Y.Y., Tang, S.Y., Zhao, Q.Z., Zhang, Z.H., Zhang, H.W. Dong, L., Guo, H.S. and Xie, Q. (2010) An efficient system to detect protein ubiquitination by agroinfiltration in Nicotiana benthamiana. Plant $\mathrm{J}$. 61, 893-903.

Liu, Z.Q., Yan, L., Wu, Z., Mei, C., Lu, K., Yu, Y.T., Liang, S., Zhang, X.F., Wang, X.F. and Zhang, D.P. (2012) Cooperation of three WRKY-domain transcription factors WRKY18, WRKY40, and WRKY60 in repressing two ABA-responsive genes $\mathrm{ABI} 4$ and $\mathrm{ABI} 5$ in Arabidopsis. J. Exp. Bot. 63 6371-6392.

Lu, F.L., Cui, X., Zhang, S.B., Jenuwein, T. and Cao, X.F. (2011) Arabidopsis REF6 is a histone H3 lysine 27 demethylase. Nat. Genet. 43, 715-719.

Magome, H., Yamaguchi, S., Hanada, A., Kamiya, Y. and Oda, K. (2008) The DDF1 transcriptional activator upregulates expression of a gibberellindeactivating gene, GA20x7, under high-salinity stress in Arabidopsis. Plant J. 56, 613-626.

Murase, K., Hirano, Y., Sun, T.P. and Hakoshima, T. (2008) Gibberellininduced DELLA recognition by the gibberellin receptor GID1. Nature 456, 459-U415.

Nambara, E. and Marion-Poll, A. (2005) Abscisic acid biosynthesis and catabolism. Annu. Rev. Plant Biol. 56, 165-185.

Nelissen, H., Rymen, B., Jikumaru, Y., Demuynck, K., Van Lijsebettens, M. Kamiya, Y., Inze, D. and Beemster, G.T. (2012) A local maximum in gibberellin levels regulates maize leaf growth by spatial control of cell division. Curr. Biol. 22, 1183-1187.

Oh, E., Yamaguchi, S., Hu, J.H., Yusuke, J., Jung, B., Paik, I., Lee, H.S., Sun T.P., Kamiya, Y. and Choi, G. (2007) PIL5, a phytochrome-interacting bHLH protein, regulates gibberellin responsiveness by binding directly to the GAI and RGA promoters in Arabidopsis seeds. Plant Cell, 19, 11921208.

Penfield, S., Li, Y., Gilday, A.D., Graham, S. and Graham, I.A. (2006) Ara bidopsis ABA INSENSITIVE4 regulates lipid mobilization in the embryo and reveals repression of seed germination by the endosperm. Plant Cell, 18, 1887-1899.

Peng, J., Carol, P., Richards, D.E., King, K.E., Cowling, R.J., Murphy, G.P. and Harberd, N.P. (1997) The Arabidopsis GAl gene defines a signaling pathway that negatively regulates gibberellin responses. Genes Dev. 11, 3194-3205.

Piskurewicz, U. and Lopez-Molina, L. (2009) The GA-signaling repressor RGL3 represses testa rupture in response to changes in GA and $A B A$ levels. Plant Signal Behav. 4, 63-65. 
Porri, A., Torti, S., Romera-Branchat, M. and Coupland, G. (2012) Spatially distinct regulatory roles for gibberellins in the promotion of flowering of Arabidopsis under long photoperiods. Development, 139, 2198-2209.

Quesada, V., Ponce, M.R. and Micol, J.L. (2000) Genetic analysis of salt-tolerant mutants in Arabidopsis thaliana. Genetics, 154, 421-436.

Reyes, J.L. and Chua, N.H. (2007) ABA induction of miR159 controls transcript levels of two MYB factors during Arabidopsis seed germination. Plant J. 49, 592-606.

Rieu, I., Eriksson, S., Powers, S.J. et al. (2008a) Genetic analysis reveals that C-19-GA 2-oxidation is a major gibberellin inactivation pathway in Arabidopsis. Plant Cell, 20, 2420-2436.

Rieu, I., Ruiz-Rivero, O., Fernandez-Garcia, N. et al. (2008b) The gibberellin biosynthetic genes AtGA20ox1 and AtGA20ox2 act, partially redundantly, to promote growth and development throughout the Arabidopsis life cycle. Plant J. 53, 488-504.

Rymen, B. and Sugimoto, K. (2012) Tuning growth to the environmental demands. Curr. Opin. Plant Biol. 15, 683-690.

Schomburg, F.M., Bizzell, C.M., Lee, D.J., Zeevaart, J.A.D. and Amasino, R.M. (2003) Overexpression of a novel class of gibberellin 2-oxidases decreases gibberellin levels and creates dwarf plants. Plant Cell, 15, 151163.

Seo, M., Hanada, A., Kuwahara, A. et al. (2006) Regulation of hormone metabolism in Arabidopsis seeds: phytochrome regulation of abscisic acid metabolism and abscisic acid regulation of gibberellin metabolism. Plant J. 48, 354-366.

Shang, Y., Yan, L., Liu, Z.Q. et al. (2010) The Mg-Chelatase H subunit of Arabidopsis Antagonizes a group of WRKY transcription repressors to relieve ABA-responsive genes of inhibition. Plant Cell, 22, 1909-1935.

Shinozaki, K. and Yamaguchi Shinozaki, K. (1997) Gene expression and signal transduction in water-stress response. Plant Physiol. 115, 327-334.

Shkolnik-Inbar, D. and Bar-Zvi, D. (2010) ABI4 mediates abscisic acid and cytokinin inhibition of lateral root formation by reducing polar auxin transport in Arabidopsis. Plant Cell, 22, 3560-3573.

Shu, K., Zhang, H., Wang, S., Chen, M., Wu, Y., Tang, S., Liu, C., Feng, Y. Cao, X. and Xie, $\mathbf{0}$. (2013) ABI4 regulates primary seed dormancy by regulating the biogenesis of abscisic acid and gibberellins in arabidopsis. PLoS Genet. 9, e1003577.

Shu, K., Wu, Y., Yang, W. and Xie, Q. (2014) Concurrent deficiency of gibberellins and abscisic Acid causes plant male sterility. J. Genet. Genomics, 41, 601-604.

Soderman, E.M., Brocard, I.M., Lynch, T.J. and Finkelstein, R.R. (2000) Regulation and function of the arabidopsis ABA-insensitive4 gene in seed and abscisic acid response signaling networks. Plant Physiol. 124, 1752-1765.

Stalberg, K., Ellerstrom, M., Josefsson, L.G. and Rask, L. (1993) Deletion analysis of a $2 \mathrm{~S}$ seed storage protein promoter in Brassica napus in transgenic tobacco. Plant Mol. Biol. 23, 671-683.

Sun, X.W., Feng, P.O., Xu, X.M., Guo, H.L., Ma, J.F., Chi, W., Lin, R.C., Lu, C.M. and Zhang, L.X. (2011) A chloroplast envelope-bound PHD transcription factor mediates chloroplast signals to the nucleus. Nature Commun. 2, 477.
Thompson, A.J., Jackson, A.C., Symonds, R.C., Mulholland, B.J., Dadswell, A.R., Blake, P.S., Burbidge, A. and Taylor, I.B. (2000) Ectopic expression of a tomato 9-cis-epoxycarotenoid dioxygenase gene causes over-production of abscisic acid. Plant J. 23, 363-374.

Toh, S., Imamura, A., Watanabe, A. et al. (2008) High temperature-induced abscisic acid biosynthesis and its role in the inhibition of gibberellin action in Arabidopsis seeds. Plant Physiol. 146, 1368-1385.

Tong, Z., Hong, B., Yang, Y.J., Li, Q.H., Ma, N., Ma, C. and Gao, J.P. (2009) Overexpression of two chrysanthemum DgDREB1 group genes causing delayed flowering or dwarfism in Arabidopsis. Plant Mol. Biol. 71, 115 129

Ueguchi-Tanaka, M., Ashikari, M., Nakajima, M. et al. (2005) GIBBERELLIN INSENSITIVE DWARF1 encodes a soluble receptor for gibberellin. Nature, 437, 693-698.

Vanstraelen, M. and Benkova, E. (2012) Hormonal interactions in the regulation of plant development. Annu. Rev. Cell Dev. Biol. 28, 463-487.

Willige, B.C., Ghosh, S., Nill, C., Zourelidou, M., Dohmann, E.M.N., Maier, A. and Schwechheimer, C. (2007) The DELLA domain of GA INSENSITIVE mediates the interaction with the GA INSENSITIVE DWARF1A gibberellin receptor of Arabidopsis. Plant Cell, 19, 1209-1220.

Wind, J.J., Peviani, A., Snel, B., Hanson, J. and Smeekens, S.C. (2013) ABI4: versatile activator and repressor. Trends Plant Sci., 18, 125-132.

Xiong, L., Ishitani, M., Lee, H. and Zhu, J.K. (2001) The Arabidopsis LOS5/ ABA3 locus encodes a molybdenum cofactor sulfurase and modulates cold stress- and osmotic stress-responsive gene expression. Plant Cell, 13, 2063-2083.

Yaish, M.W., El-kereamy, A., Zhu, T., Beatty, P.H., Good, A.G., Bi, Y.M. and Rothstein, S.J. (2010) The APETALA-2-Like transcription factor OsAP2-39 controls key interactions between abscisic acid and gibberellin in rice. PLoS Genet. 6, e1001098.

Yamaguchi, S. (2008) Gibberellin metabolism and its regulation. Ann. Rev. Plant Biol. 59, 225-251.

Yamauchi, Y., Ogawa, M., Kuwahara, A., Hanada, A., Kamiya, Y. and Yamaguchi, S. (2004) Activation of gibberellin biosynthesis and response pathways by low temperature during imbibition of Arabidopsis thaliana seeds. Plant Cell, 16, 367-378.

Yano, R., Kanno, Y., Jikumaru, Y., Nakabayashi, K., Kamiya, Y. and Nambara, E. (2009) CHOTTO1, a putative double APETALA2 repeat transcription factor, is involved in abscisic acid-mediated repression of Gibberellin Biosynthesis during seed germination in Arabidopsis. Plant Physiol. 151, 641-654

Zentella, R., Zhang, Z.L., Park, M. et al. (2007) Global analysis of DELLA direct targets in early gibberellin signaling in Arabidopsis. Plant Cell, 19, 3037-3057.

Zhang, Z., Chen, H., Huang, X. et al. (2011a) BSCTV C2 attenuates the degradation of SAMDC1 to suppress DNA methylation-mediated gene silencing in Arabidopsis. Plant Cell, 23, 273-288.

Zhang, Z.L., Ogawa, M., Fleet, C.M., Zentella, R., Hu, J.H., Heo, J.O., Lim, J., Kamiya, Y., Yamaguchi, S. and Sun, T.P. (2011b) SCARECROW-LIKE 3 promotes gibberellin signaling by antagonizing master growth repressor DELLA in Arabidopsis. Proc. Natl Acad. Sci. USA, 108, 2160-2165. 